\title{
Some perturbation results of Kirchhoff type equations via Morse theory
}

\author{
Mingzheng Sun ${ }^{1}$, Yutong Chen ${ }^{2}$ and Rushun Tian ${ }^{2 *}$ (])
}

\begin{tabular}{l}
\hline${ }^{*}$ Correspondence: \\
rushun.tian@cnu.edu.cn \\
${ }^{2}$ School of Mathematical Sciences, \\
Capital Normal University, 100048, \\
Beijing, China \\
Full list of author information is \\
available at the end of the article
\end{tabular}

available at the end of the article

\begin{abstract}
In this paper, we consider the following Kirchhoff type equation:

$$
\begin{cases}-\left(a+b \int_{\Omega}|\nabla u|^{2} d x\right) \Delta u=f(x, u) & \text { in } \Omega, \\ u=0 & \text { on } \partial \Omega\end{cases}
$$

where $a, b>0$ are constants and $\Omega \subset \mathbb{R}^{N}(N=1,2,3)$ is a bounded domain with smooth boundary $\partial \Omega$. By applying Morse theory, we obtain some existence and multiplicity results of nontrivial solutions for either $a$ or $b$ being sufficiently small.
\end{abstract}

MSC: $35 \mathrm{~J} 65 ; 35 J 20 ; 47 J 10$

Keywords: Kirchhoff equations; Nontrivial solutions; Morse theory

\section{Introduction}

In this paper, we are concerned with the Kirchhoff equation,

$$
u_{t t}-\left(a+b \int_{\Omega}|\nabla u|^{2} d x\right) \Delta u=f(x, u)
$$

which was proposed by Kirchhoff in [13] as a generalization of the well-known d'Alembert wave equation

$$
\rho \frac{\partial^{2} u}{\partial t^{2}}-\left(\frac{\rho_{0}}{h}+\frac{E}{2 L} \int_{0}^{L}\left|\frac{\partial u}{\partial x}\right|^{2} d x\right) \frac{\partial^{2} u}{\partial x^{2}}=0
$$

for free vibrations of elastic strings, where $\rho$ is the mass density, $\rho_{0}$ is the initial tension, $h$ is the area of the cross section, $E$ is the Young modulus of the material and $L$ is the length of the string. Kirchhoff's model takes the changes in length of the string produced by transverse vibrations into account. We refer to $[13,18]$ for further references in physics.

The stationary analogue of the Kirchhoff equation takes the form

$$
\begin{cases}-\left(a+b \int_{\Omega}|\nabla u|^{2} d x\right) \Delta u=f(x, u) & \text { in } \Omega, \\ u=0 & \text { on } \partial \Omega\end{cases}
$$

(c) The Author(s) 2020. This article is licensed under a Creative Commons Attribution 4.0 International License, which permits use, sharing, adaptation, distribution and reproduction in any medium or format, as long as you give appropriate credit to the original author(s) and the source, provide a link to the Creative Commons licence, and indicate if changes were made. The images or other third party material in this article are included in the article's Creative Commons licence, unless indicated otherwise in a credit line to the material. If material is not included in the article's Creative Commons licence and your intended use is not permitted by statutory regulation or exceeds the permitted use, you will need to obtain permission directly from the copyright holder. To view a copy of this licence, visit http://creativecommons.org/licenses/by/4.0/. 
where $a, b>0$ are constants, and $\Omega$ is a bounded domain in $\mathbb{R}^{N}(N=1,2,3)$ with smooth boundary $\partial \Omega$. We are interested in the case that $f$ is sub-critical, i.e.

$\left(f_{0}\right) f \in \mathcal{C}(\bar{\Omega} \times \mathbb{R}, \mathbb{R})$ and there exists $c>0$ such that

$$
|f(x, u)| \leq c\left(1+|u|^{\gamma-1}\right), \quad \text { for some } 1 \leq \gamma<2^{*}= \begin{cases}+\infty, & N=1,2 \\ 6, & N=3 .\end{cases}
$$

The weak solutions of (1.1) then correspond to critical points of $I_{(a, b)}: H_{0}^{1}(\Omega) \rightarrow \mathbb{R}$,

$$
I_{(a, b)}(u)=\frac{a}{2}\|u\|^{2}+\frac{b}{4}\|u\|^{4}-\int_{\Omega} F(x, u) d x
$$

where $F(x, u)=\int_{0}^{u} f(x, t) d t$, and $H_{0}^{1}(\Omega)$ is the Sobolev space endowed with the norm

$$
\|u\|=\|\nabla u\|_{2}=\left(\int_{\Omega}|\nabla u|^{2} d x\right)^{1 / 2} .
$$

To characterize the growth rate of $f(x, t)$ at $t=0$, we consider two eigenvalue problems. First, let

$$
0<\lambda_{1}<\lambda_{2} \leq \cdots \leq \lambda_{m} \leq \cdots
$$

be the sequences of eigenvalues (counted with multiplicity) of $-\Delta$ in $\Omega$ with Dirichlet boundary condition. Second, according to [23], the nonlocal eigenvalue problem

$$
-\|\phi\|^{2} \Delta \phi=\mu \phi^{3} \quad \text { in } \Omega, \quad \phi=0 \quad \text { on } \partial \Omega,
$$

has an unbounded sequences of eigenvalues, counted with multiplicities

$$
0<\mu_{1} \leq \mu_{2} \leq \cdots \leq \mu_{m} \leq \cdots
$$

Denote by $\lambda_{0}=\mu_{0}=-\infty$ and by $\Sigma$ the set of all the eigenvalues of (1.3).

Now we introduce two growth conditions for $f(x, u)$ :

(GC1) there exists $\mu \in\left(\lambda_{m}, \lambda_{m+1}\right)$ such that $\lim _{|u| \rightarrow 0} \frac{f(x, u)}{u}=a \mu$, uniformly in $x \in \Omega$;

(GC3) there exists $\mu \in\left(\mu_{m}, \mu_{m+1}\right) \backslash \Sigma$ such that $\lim _{|u| \rightarrow 0} \frac{f(x, u)}{u^{3}}=b \mu$, uniformly in $x \in \Omega$, where $a, b$ are the constants appear in Eq. (1.1). Clearly, both (GC1) and (GC3) imply $f(x, 0) \equiv 0$ so problem (1.1) admits a trivial solution $u=0$. We are interested in the existence of nontrivial solutions.

In recent years, many papers study the Kirchhoff type problems by variational methods. When the nonlinearity is 4-superlinear near infinity, the relevant results can be found in $[20,24,25,32]$, and for the case where the nonlinearity is 4-asymptotically linear near infinity, we refer to $[8,15,17,23,30,32]$ for details and further references. For example, if (GC1) holds with $\mu<\lambda_{1}$, then it is shown in [8] that 0 is a local minimizer of $I_{(a, b)}$. With the condition

$$
\frac{a}{2} \lambda_{k} t^{2}+C_{1} t^{4} \leq F(x, t) \leq \frac{a}{2} \lambda_{k+1} t^{2}+C_{2} t^{4}, \quad \text { for }|t|<\delta,
$$


where $\delta, C_{1}, C_{2}$ are positive constants, it is shown in [25] that the functional $I_{(a, b)}$ has a local linking at zero. Also, using the sequence of eigenvalues constructed in [23], the authors of [24] find nontrivial solutions when the nonlinearity is superlinear near zero but asymptotically 4 -linear at infinity by computing the relevant critical groups.

In particular, when the nonlinearity is concave-convex, that is,

$$
f(x, u)=\lambda g_{1}(x)|u|^{q-2} u+g_{2}(x)|u|^{p-2} u,
$$

for $\lambda>0,1<q<2<p<2^{*}$ and possibly sign-changing functions $g_{1}(x), g_{2}(x) \in \mathcal{C}(\Omega)$, by the Nehari manifold and fibering maps, the existence of multiple positive solutions is established in [7]. In [14], a power-type concave-convex nonlinearity with critical exponent is considered, and two positive solutions are found for $b$ being small. For a nonhomogeneous $p$-Kirchhoff-type equation with nonlinearity as (1.5) in unbounded domains, the existence of multiple solutions for problem (1.1) is studied in [6], by Ekeland's variational principle and the mountain pass theorem. Moreover, without any growth condition on the nonlinear term $f$ at infinity, the paper [28] obtain a sequence of solutions converging to zero for Kirchhoff equation with local sublinear nonlinearities. For more details about the existence of solutions for Kirchhoff-type equation involving concave and convex terms, we refer to $[10,16,29]$ for details and further references. Furthermore, problem (1.1) can be generalized to $p$-Kirchoff equations and fractional Kirchoff equations. For instance, fractional Kirchoff equations in the case $\Omega=\mathbb{R}$ have been studied in [22, 27], in which the Morse theory were applied to obtain multiple nontrivial solutions. We also refer to $[2,3]$ by Chang for a systematic introduction of Morse theory and various applications to differential equations.

Notice that the parameters $a>0$ and $b>0$ are fixed in all papers cited above. The parameters $a$ and $b$ affect the nature of the equation in the following way. If $a>0$, Eq. (1.1) is said to be non-degenerate; and it is called degenerate if $a=0$ (see e.g., [9, 31]). On the other hand, if $b=0,(1.1)$ is a usual Laplacian equation. If $b>0$, Eq. (1.1) becomes a nonlocal, i.e., Eq. (1.1) is no longer a pointwise equality. This nonlocal nature causes some mathematical difficulties which make the study of such problems particularly interesting. Then it seems rather natural to ask whether it is possible to get some relationships between the two solutions for equations with $a=0$ and $b=0$, respectively. Motivated by the methods in $[5,26]$, we will give some answer to this question through the estimates of critical groups for critical points of functionals, and we also use Morse theory to obtain the existence of nontrivial solutions of (1.1).

Our results read as follows.

Theorem 1.1 Let $b>0$ be fixed. Iff satisfies $\left(f_{0}\right)$ and (GC3) with $m \geq 1$, then there exists $\varepsilon>0$ such that, for each $a \in(0, \varepsilon), E q$. (1.1) has at least one nontrivial solution.

\section{Remark 1}

(1) Notice that $f(x, u)=b \mu u^{3}$ for some $\mu \in\left(\mu_{m}, \mu_{m+1}\right)$ satisfies conditions $\left(f_{0}\right)$ and (GC3), then (1.1) becomes

$$
-\left(a+b\|u\|^{2}\right) \Delta u=b \mu u^{3} \quad \text { in } \Omega \text { and } u=0 \text { on } \partial \Omega .
$$


For any $a>0$, the existence of a nontrivial solution has been proved in [23, Theorem 1.1].

(2) The main novelty of Theorem 1.1 is that no additional assumption on the nonlinearity $f$ near infinity besides $\left(f_{0}\right)$ is required. In comparison, the behavior of $f$ near infinity is used in an essential way to get the compactness condition, or derive multiplicity of solutions in the papers we quoted previously. Moreover, let $f \in \mathcal{C}(\mathbb{R})$ be a function of $u$, and $f(u)=b \mu u^{3}$ for $|u|<1$, and $f(u)=|u|^{p-2} u$ for $|u|>2$, $1 \leq p<2^{*}$. Since $f$ may take different forms for $1<|u|<2$, Theorem 1.1 asserts the existence of nontrivial solution for nonlinearities not only restricted to power-type, which in contrast plays an important role for applying Nehari manifold type arguments.

(3) Denote by $C_{\ell}\left(I_{(a, b)}, u\right)$ the $\ell$ th critical group of the $C^{1}$ functional $I_{(a, b)}$ at an isolated critical point $u$ (see precise definition in Sect. 2), where $l \in \mathbb{N}=\{0,1,2, \ldots\}$. The key step in the proof of Theorem 1.1 is to deduce the facts that $u=0$ is an isolated critical point of $I_{(0, b)}$ and its critical group $C_{m}\left(I_{(0, b)}, 0\right)$ is nontrivial.

(4) If $f$ satisfies $\left(f_{0}\right)$ and (GC1) with $m \geq 0$, then $u=0$ is also an isolated critical point of $I_{(a, 0)}$ and $C_{m}\left(I_{(a, 0)}, 0\right)$ is nontrivial (see [2]). But the approach to proving Theorem 1.1 is no longer able to guarantee the existence of a nontrivial solution of (1.1).

From the arguments in the proof of Theorem 1.1, we can deduce the following results.

\section{Corollary 1.1 Assume that $\left(f_{0}\right)$ holds.}

(i) Let $a>0$ be fixed. If $u_{0}$ is an isolated critical point of $I_{(a, 0)}$ with its critical group $C_{\ell}\left(I_{(a, 0)}, u_{0}\right)$ nontrivial at some $\ell \in \mathbb{N}$. Then there exists $\varepsilon>0$ such that, for each $b \in(0, \varepsilon), E q .(1.1)$ has at least a solution $u_{\varepsilon}$.

(ii) Let $b>0$ be fixed. If $u_{0}$ is an isolated critical point of $I_{(0, b)}$ with its critical group $C_{\ell}\left(I_{(0, b)}, u_{0}\right)$ nontrivial at some $\ell \in \mathbb{N}$. Then there exists $\varepsilon>0$ such that, for each $a \in(0, \varepsilon), E q$. (1.1) has at least a solution $u_{\varepsilon}$.

In both cases, there exists a sequence $\left\{\varepsilon_{n}\right\}$ and corresponding $\left\{u_{\varepsilon_{n}}\right\}$ such that $\varepsilon_{n} \rightarrow 0$ and $u_{\varepsilon_{n}} \rightarrow u_{0}$ in $H_{0}^{1}(\Omega)$ as $n \rightarrow \infty$.

Next, we consider nonlinearity $f$ with a perturbation term,

$$
f(x, u)=\lambda|u|^{p-2} u+g(x, u)
$$

where $1<p<6$ and $\lambda \in \mathbb{R}$. Clearly, if $\lambda=0$, then it returns to the case in Theorem 1.1.

Theorem 1.2 Assume $1<p<2$ and $m \geq \frac{1}{2}(1-\operatorname{sgn}(\lambda))$. If $g$ satisfies $\left(f_{0}\right)$ (i.e. replacing $f$ with $g$ in $\left.\left(f_{0}\right)\right)$, then there exists $\varepsilon>0$ such that Eq. (1.1) has at least one nontrivial solution in either of the following cases:

(i) $0<a,|\lambda|<\varepsilon, b>0$ is fixed and $g$ satisfies (GC3);

(ii) $0<b,|\lambda|<\varepsilon, a>0$ is fixed and $g$ satisfies (GC1).

Remark 2 In particular, if $g(x, u)=b \mu u^{3}$, then the existence of nontrivial solution was proved in [7], which provides an example for Theorem 1.2 (i). Moreover, as pointed out in Remark 1 (2), $g$ may take other than power-type forms, thus Theorem 1.2 and Theorem 1.3 are both new for dealing with such nonlinearities. 
Theorem 1.3 Assume $2 \leq p<6$ and $m \geq 1$. If $g$ satisfies $\left(f_{0}\right)$ and (GC3) with $b>0$ is fixed, then there exists $\varepsilon>0$ such that, for $0<a,|\lambda|<\varepsilon, E q$. (1.1) has at least one nontrivial solution.

This paper is organized as follows. In Sect. 2, we will recall some established results of Morse theory. In Sect. 3, we give the proofs of Theorem 1.1 and Corollary 1.1. The proofs of Theorem 1.2 and Theorem 1.3 are given in Sects. 4 and 5, respectively. In the sequel, the letter $C$ will be used indiscriminately to denote a suitable positive constant whose value may change from line to line.

\section{Preliminaries}

In this section, we summarize some well known results that will be used in later sections.

Let $I$ be a $\mathcal{C}^{1}$ functional defined on a Banach space $X$, and denote the set of critical points of $I$ by $K_{I}$. We also assume that $I$ satisfies the Palais-Smale condition. We shall prove the existence of multiple solutions by contradiction, for which the trivial solution will be assumed to be isolated at first. Then to apply the Morse theory, the critical group of isolated critical points needs to be generalized to critical group of compact dynamically isolated critical set using the Gromoll-Meyer pair. Precisely,

Proposition 2.1 ([2, Theorem 5.2 and 5.3 in Chapter I]) If $S=\left\{u_{0}\right\}$, where $u_{0}$ is an isolated critical point of $I$, then there exists a Gromoll-Meyer pair $\left(W, W_{-}\right)$for $S=\left\{u_{0}\right\}$ such that

$$
C_{\ell}\left(I, u_{0}\right)=C_{\ell}(I,[S])=H_{\ell}\left(W, W_{-}\right), \quad \ell \in \mathbb{N} .
$$

Here $[S]$ denotes an invariant hull of $S$, and if $u_{0}$ is an isolated critical point that is located on an isolated critical level, then the singleton $S=\left\{u_{0}\right\}$ is a dynamically isolated critical set, and $[S]=S=\left\{u_{0}\right\}$.

The following proposition is crucial in applying the perturbation type arguments.

Proposition 2.2 ([4, Theorem III.4]) Let $S$ be a compact dynamically isolated critical set for the functional I, and $\left(W, W_{-}\right)$is a Gromoll-Meyer pair for $S$. Then there exists $\varepsilon>0$ depending on $I$ and $W$ such that, for all $J \in \mathcal{C}^{1}(X, \mathbb{R})$ with $\|I-J\|_{\mathcal{C}^{1}(W)}<\varepsilon,\left(W, W_{-}\right)$is still a Gromoll-Meyer pair for the critical set $S_{J}=W \cap K_{J}$.

The homotopy invariance of critical group also plays an important role in our proofs.

Proposition $2.3([4])$ Let $I_{\tau} \in C^{1}(X)$ and $u_{0} \in K_{I_{\tau}}$ for all $\tau \in[0,1]$. If there exists a closed neighborhood $U \subset X$ of $u_{0}$ such that

(i) $I_{\tau}$ satisfies the Palais-Smale condition in $U$ for all $\tau \in[0,1]$,

(ii) $K_{I_{\tau}} \cap U=\left\{u_{0}\right\}$ for all $\tau \in[0,1]$,

(iii) the mapping $\tau \rightarrow I_{\tau}$ is continuous between $[0,1]$ and $C^{1}(U)$, then we have

$$
C_{\ell}\left(I_{0}, u_{0}\right)=C_{\ell}\left(I_{1}, u_{0}\right), \quad \ell \in \mathbb{N}
$$


Finally, for any given $\mu \in \mathbb{R}$, define $\Phi: H_{0}^{1}(\Omega) \rightarrow \mathbb{R}$ by

$$
\Phi(u)=\|u\|^{4}-\mu \int_{\Omega} u^{4} d x
$$

If $\mu \notin \Sigma$, then $u=0$ is an isolated critical point of $\Phi$. Therefore $C_{*}(\Phi, 0)$, the critical groups of $\Phi$ at 0 , are well-defined; see [3]. The following result was proved by Perera and Zhang in [23].

Proposition 2.4 ([23, Proposition 3.3]) If $\mu \in\left(\mu_{m}, \mu_{m+1}\right) \backslash \Sigma$, then $C_{m}(\Phi, 0) \neq 0$.

\section{Proof of Theorem 1.1}

We begin with a few lemmas.

Lemma 3.1 Assume that $\left(f_{0}\right)$ and (GC3) hold, then $u=0$ is an isolated critical point of $I_{(0, b)}$.

Proof The proof is partially inspired by [11]. Note that $I_{(0, b)}$ is a $C^{1}$ functional and

$$
\left\langle I_{(0, b)}{ }^{\prime}(u), w\right\rangle=b\|u\|^{2} \int_{\Omega} \nabla u \nabla w d x-\int_{\Omega} f(x, u) w d x, \quad \forall w \in H_{0}^{1}(\Omega) .
$$

Clearly, by $f(x, 0)=0$ we have $u=0$ is a critical point of $I_{(0, b)}$.

If the conclusion is not true, then there exists a sequence $\left\{u_{n}\right\} \subset H_{0}^{1}(\Omega) \backslash\{0\}$ such that

$$
u_{n} \rightarrow 0 \quad \text { in } H_{0}^{1}(\Omega) \quad \text { and } \quad I_{(0, b)}{ }^{\prime}\left(u_{n}\right)=0 \quad \text { for any } n \in \mathbb{N} .
$$

Set $v_{n}=u_{n} /\left\|u_{n}\right\|$, then $\left\|v_{n}\right\|=1$. Passing to a subsequence we can assume

$$
\begin{cases}v_{n} \rightarrow v, & \text { weakly in } H_{0}^{1}(\Omega), \\ v_{n} \rightarrow v, & \text { strongly in } L^{4}(\Omega), \\ v_{n}(x) \rightarrow v(x), & \text { a.e. } x \in \Omega,\end{cases}
$$

as $n \rightarrow \infty$. Define

$$
\xi_{n}(x)= \begin{cases}\frac{f\left(x, u_{n}\right)}{b u_{n}^{3}}, & u_{n}(x) \neq 0, \\ \mu, & u_{n}(x)=0,\end{cases}
$$

then $\xi_{n} \rightarrow \mu$ a.e. in $\Omega$ as $n \rightarrow \infty$ by (GC3).

Moreover, we have

$$
\begin{aligned}
0 & =\frac{\left\langle I_{(0, b)}{ }^{\prime}\left(u_{n}\right), w\right\rangle}{b\left\|u_{n}\right\|^{3}} \\
& =\int_{\Omega} \nabla v_{n} \nabla w d x-\int_{\Omega} \xi_{n}(x) v_{n}^{3} w d x+\int_{\Omega}\left(\xi_{n}(x) v_{n}^{3}-\frac{f\left(x, u_{n}\right)}{b\left\|u_{n}\right\|^{3}}\right) w d x,
\end{aligned}
$$

this together with (GC3) gives

$$
\int_{\Omega} \nabla v \nabla w d x=\mu \int_{\Omega} v^{3} w d x
$$


Replace $w$ with $v_{n}-v$ in (3.2) and let $n \rightarrow \infty$, we get $\|v\|=1$. Then (3.3) implies that $\mu$ is an eigenvalue of (1.3), which is a contradiction since $\mu \in\left(\mu_{m}, \mu_{m+1}\right) \backslash \Sigma$. The proof is completed.

Lemma 3.2 Assume that $\left(f_{0}\right)$ and (GC3) hold, then

$$
C_{\ell}(\Phi, 0)=C_{\ell}\left(I_{(0, b)}, 0\right), \quad \ell \in \mathbb{N} .
$$

Proof Define $J_{t}: H_{0}^{1}(\Omega) \rightarrow \mathbb{R}$ as

$$
J_{t}(u)=t I_{(0, b)}(u)+\frac{(1-t) b}{4} \Phi(u), \quad t \in[0,1]
$$

then by $\left(f_{0}\right) J_{t}$ satisfies $(P S)$ condition on any bounded domain in $H_{0}^{1}(\Omega)$ for $t \in[0,1]$. Clearly, $u=0$ is a critical point for all $t \in[0,1]$. If we can find a neighborhood $U$ of 0 such that $u=0$ is the only critical point of $J_{t}$ in $U$ for all $t \in[0,1]$, then by the homotopy invariance of the critical groups in Proposition 2.3 we have

$$
C_{\ell}(\Phi, 0)=C_{\ell}\left(J_{0}, 0\right)=C_{\ell}\left(J_{1}, 0\right)=C_{\ell}\left(I_{(0, b)}, 0\right), \quad \ell \in \mathbb{N} .
$$

We argue by contradiction. Assume that there exist sequences $\left\{t_{n}\right\} \subset[0,1]$ and $\left\{u_{n}\right\} \subset$

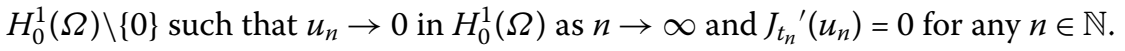

Set $v_{n}=u_{n} /\left\|u_{n}\right\|$, and passing to a subsequence we may assume that $t_{n} \rightarrow t_{0}$ and

$$
\begin{cases}v_{n} \rightarrow v, & \text { weakly in } H_{0}^{1}(\Omega), \\ v_{n} \rightarrow v, & \text { strongly in } L^{4}(\Omega), \\ v_{n}(x) \rightarrow v(x), & \text { a.e. } x \in \Omega,\end{cases}
$$

as $n \rightarrow \infty$. Similar to (3.2), define $\xi_{n}(x)$ as (3.1), we get

$$
\begin{aligned}
0= & \frac{\left\langle t_{n}{ }^{\prime}\left(u_{n}\right), w\right\rangle}{b\left\|u_{n}\right\|^{3}} \\
= & \int_{\Omega}\left[\nabla v_{n} \nabla w-\left(1-t_{n}\right) \mu v_{n}^{3} w-t_{n} \xi_{n}(x) v_{n}^{3} w\right] d x \\
& +t_{n} \int_{\Omega}\left(\xi_{n}(x) v_{n}^{3}-\frac{f\left(x, u_{n}\right)}{b\left\|u_{n}\right\|^{3}}\right) w d x \\
= & \int_{\Omega}\left[\nabla v_{n} \nabla w-\mu v_{n}^{3} w+t_{n}\left(\mu-\xi_{n}(x)\right) v_{n}^{3} w\right] d x \\
& +t_{n} \int_{\Omega}\left(\xi_{n}(x) v_{n}^{3}-\frac{f\left(x, u_{n}\right)}{b\left\|u_{n}\right\|^{3}}\right) w d x .
\end{aligned}
$$

Let $n \rightarrow \infty$ and recall the condition (GC3), we have

$$
\int_{\Omega} \nabla v \nabla w d x=\mu \int_{\Omega} v^{3} w d x, \quad \forall w \in H_{0}^{1}(\Omega)
$$

Again, replacing $w$ with $v_{n}-v$ in (3.4) we get $\|v\|=1$, which implies that $\mu$ is an eigenvalue of (1.3). This contradicts the assumption. The proof is completed. 
Combining Proposition 2.4 and Lemma 3.2, we obtain the following.

Lemma 3.3 Assume $\left(f_{0}\right)$ and (GC3) hold, then

$$
C_{m}\left(I_{(0, b)}, 0\right) \neq 0 .
$$

By introducing the quadratic term, the critical group at zero will change.

Lemma 3.4 Assume that $f$ satisfies $\left(f_{0}\right)$ and (GC3), then we have

$$
C_{\ell}\left(I_{(a, b)}, 0\right)=\delta_{\ell, 0} \mathbb{F}, \quad \ell \in \mathbb{N},
$$

where $\delta$ is the Kronecker delta.

Proof In fact, by $\left(f_{0}\right)$ and (GC3), there exist $C>0$ and $\gamma \in\left(2,2^{*}\right)$ such that

$$
\int_{\Omega} F(x, u) d x \leq C\|u\|^{4}+C\|u\|^{\gamma}
$$

hence, for $\|u\|>0$ small enough,

$$
\begin{aligned}
I_{(a, b)}(u) & =\frac{a}{2}\|u\|^{2}+\frac{b}{4}\|u\|^{4}-\int_{\Omega} F(x, u) d x \\
& \geq \frac{a}{2}\|u\|^{2}+\frac{b}{4}\|u\|^{4}-C\|u\|^{4}-C\|u\|^{\gamma} \\
& \geq 0 .
\end{aligned}
$$

So 0 is a local minimizer of $I_{(a, b)}$, and this lemma is true.

Proof of Theorem 1.1 Note that, by $\left(f_{0}\right)$, our functionals satisfy the Palais-Smale condition on any closed bounded set. The proof is divided into four steps.

(1) By Lemma 3.1, $u=0$ is an isolated critical point of $I_{(0, b)}$. Without loss of generality, we may assume that $u=0$ is the only critical point of $I_{(0, b)}$ in the ball

$$
B_{\rho}(0)=\left\{u \in H_{0}^{1}(\Omega):\|u\|<\rho\right\}, \quad \text { for some } \rho>0 .
$$

Proposition 2.1 implies that there exists a Gromoll-Meyer pair $\left(W, W_{-}\right)$for $I_{(0, b)}$ at 0 satisfying $W \subset B_{\rho}(0)$ such that

$$
C_{\ell}\left(I_{(0, b)}, 0\right)=H_{\ell}\left(W, W_{-}\right), \quad \forall \ell \in \mathbb{N},
$$

this together with Lemma 3.3 gives

$$
H_{m}\left(W, W_{-}\right)=C_{m}\left(I_{(0, b)}, 0\right) \neq 0, \quad \text { for some } m \geq 1 \text {. }
$$

(2) Claim: For any $\varepsilon>0$, setting $\beta=\frac{2 \varepsilon}{\rho^{2}+2 \rho}$, then

$$
\left\|I_{(a, b)}-I_{(0, b)}\right\|_{C^{1}(W)}<\varepsilon, \quad \text { for } 0<a<\beta .
$$


Indeed, for any $v \in H_{0}^{1}(\Omega)$,

$$
\left\langle I_{(a, b)}{ }^{\prime}(u), v\right\rangle=a \int_{\Omega} \nabla u \nabla v d x+b\|u\|^{2} \int_{\Omega} \nabla u \nabla v d x-\int_{\Omega} f(x, u) v d x .
$$

Therefore

$$
\begin{aligned}
\left\|I_{(a, b)}-I_{(0, b)}\right\|_{C^{1}(W)} & =\left\|I_{(a, b)}-I_{(0, b)}\right\|_{C(W)}+\left\|I_{(a, b)}^{\prime}-I_{(0, b)}^{\prime}\right\|_{C(W)} \\
& =\sup _{u \in W}\left|I_{(a, b)}(u)-I_{(0, b)}(u)\right|+\sup _{u \in W} \sup _{\|v\| \leq 1}\left|\left\langle I_{(a, b)}^{\prime}(u)-I_{(0, b)}^{\prime}(u), v\right)\right| \\
& \leq \frac{1}{2} a \rho^{2}+a \sup _{u \in W\|v\| \leq 1}\|u\|\|v\| \\
& \leq \frac{1}{2} a\left(\rho^{2}+2 \rho\right)<\varepsilon,
\end{aligned}
$$

then (3.6) holds. Using Proposition 2.2, for $\varepsilon>0$ small enough, (3.6) implies that ( $W, W_{-}$) is still a Gromoll-Meyer pair for $I_{(a, b)}$ with the critical set

$$
S_{[a, b]}=W \cap K_{\left.I_{(a, b)}\right)}, \quad \text { for } 0<a<\beta,
$$

where

$$
K_{I_{(a, b)}}=\left\{u \in H_{0}^{1}(\Omega): I_{(a, b)}{ }^{\prime}(u)=0\right\} .
$$

Therefore, for $0<a<\beta$, using (3.5), we have

$$
C_{m}\left(I_{(a, b)},\left[S_{[a, b]}\right]\right)=H_{m}\left(W, W_{-}\right) \neq 0, \quad \text { for some } m \geq 1 \text {. }
$$

(3) Without loss of generality, we assume that $u=0$ is an isolated critical point of $I_{(a, b)}$ for $0<a<\beta$ and $b>0$. Then there exists a Gromoll-Meyer pair $\left(W^{0}, W_{-}^{0}\right)$ with $0 \in W^{0} \varsubsetneqq W$ such that

$$
C_{\ell}\left(I_{(a, b)}, 0\right)=H_{\ell}\left(W^{0}, W_{-}^{0}\right), \quad \text { for all } \ell \in \mathbb{N} .
$$

Now using Lemma 3.4, we have

$$
H_{\ell}\left(W^{0}, W_{-}^{0}\right)=C_{\ell}\left(I_{(a, b)}, 0\right)=\delta_{\ell, 0} \mathbb{F}, \quad \text { for } 0<a<\beta, b>0 .
$$

(4) Claim: $I_{(a, b)}$ has at least one nontrivial critical point in $W \backslash W^{0}$.

Assume it is not true, then $S_{[a, b]}=\{0\}$, which implies that $I_{(a, b)}$ has no critical points in $W \backslash W^{0}$. By the deformation and excision properties of a singular homology (see [2]), we may assume $W^{0}=W$ in the above choice of the Gromoll-Meyer pairs for $I_{(a, b)}$ at 0 . Therefore,

$$
H_{\ell}\left(W, W_{-}\right)=H_{\ell}\left(W^{0}, W_{-}^{0}\right), \quad \ell \in \mathbb{N},
$$

which is a contradiction by (3.7) and (3.8). Therefore, Eq. (1.1) has at least one nontrivial solution. The proof is completed. 
Proof of Corollary 1.1 Similar arguments to the proof of Theorem 1.1 yield the existence of the solution, here, we focus on the convergence of the solution.

For the case (i), let $u_{0}$ be the only critical point of $I_{(a, 0)}$ in the ball $B_{\rho}\left(u_{0}\right)$, and $u_{\varepsilon_{n}} \in B_{\rho}\left(u_{0}\right)$ be the critical point of $I_{\left(a, \varepsilon_{n}\right)}$ such that $\varepsilon_{n} \rightarrow 0$ and

$$
\left\langle I_{\left(a, \varepsilon_{n}\right)}{ }^{\prime}\left(u_{\varepsilon_{n}}\right), v\right\rangle=0, \quad \forall v \in H_{0}^{1}(\Omega)
$$

Then passing to a subsequence we may assume that

$$
\begin{cases}u_{\varepsilon_{n}} \rightarrow u^{*}, & \text { weakly in } H_{0}^{1}(\Omega), \\ u_{\varepsilon_{n}} \rightarrow u^{*}, & \text { strongly in } L^{4}(\Omega), \\ u_{\varepsilon_{n}}(x) \rightarrow u^{*}(x), & \text { a.e. } x \in \Omega\end{cases}
$$

Since $\left\langle I_{\left(a, \varepsilon_{n}\right)}{ }^{\prime}\left(u_{\varepsilon_{n}}\right)-I_{\left(a, \varepsilon_{n}\right)}{ }^{\prime}\left(u^{*}\right), u_{\varepsilon_{n}}-u^{*}\right\rangle=0$, we have, as $\varepsilon_{n} \rightarrow 0$,

$$
\begin{aligned}
\left\|u_{\varepsilon_{n}}-u^{*}\right\|^{2}= & b\left\|u^{*}\right\|^{2} \int_{\Omega} \nabla u^{*} \nabla\left(u_{\varepsilon_{n}}-u^{*}\right) d x-b\left\|u_{\varepsilon_{n}}\right\|^{2} \int_{\Omega} \nabla u_{\varepsilon_{n}} \nabla\left(u_{\varepsilon_{n}}-u^{*}\right) d x \\
& +\int_{\Omega}\left(f\left(x, u_{\varepsilon_{n}}\right)-f\left(x, u^{*}\right)\right)\left(u_{\varepsilon_{n}}-u^{*}\right) d x \\
\rightarrow & 0
\end{aligned}
$$

which implies that $u_{\varepsilon_{n}} \rightarrow u^{*}$ in $H_{0}^{1}(\Omega)$. Let $\varepsilon_{n} \rightarrow 0$ in (3.9) we get

$$
\left\langle I_{(a, 0)}{ }^{\prime}\left(u^{*}\right), v\right\rangle=0, \quad \forall v \in H_{0}^{1}(\Omega) .
$$

Then $u^{*}$ is a critical point of $I_{(a, 0)}$. But from the isolation of $u_{0}$ in $B_{\rho}\left(u_{0}\right)$, we must have $u^{*}=u_{0}$. The case (ii) is similar.

\section{Proof of Theorem 1.2}

Consider the $C^{1}$ functional $I_{(a, b, \lambda)}: H_{0}^{1}(\Omega) \rightarrow \mathbb{R}$ defined by setting

$$
I_{(a, b, \lambda)}(u)=\frac{a}{2}\|u\|^{2}+\frac{b}{4}\|u\|^{4}-\frac{\lambda}{p} \int_{\Omega}|u|^{p} d x-\int_{\Omega} G(x, u) d x,
$$

where $G(x, u)=\int_{0}^{u} g(x, t) d t$. The critical group of $I_{(a, b, \lambda)}$ at 0 can be calculated using the arguments in $[12,19,21]$. For completeness, we provide the detailed proof now.

Lemma 4.1 Assume that $g$ satisfies $\left(f_{0}\right)$ and $g(x, u)=O(|u|)$ as $|u| \rightarrow 0$, then, for any $a>0$, $b \geq 0,1<p<2$ and $\lambda>0$ we have

$$
C_{\ell}\left(I_{(a, b, \lambda)}, 0\right)=0, \quad \forall \ell \in \mathbb{N} \text {. }
$$

Proof We divide it into a few steps.

Step 1 For each $u \neq 0$, there exists a constant $s_{0}$ such that $I_{(a, b, \lambda)}(s u)<0$ for all $s \in\left(0, s_{0}\right)$. Since $g$ satisfies $\left(f_{0}\right)$ and $g(x, u)=O(|u|)$ as $|u| \rightarrow 0$, there are two constants, $\gamma \in\left(2,2^{*}\right)$ and $C>0$, such that

$$
|G(x, u)| \leq C\left(|u|^{2}+|u|^{\gamma}\right), \quad \forall u \in \mathbb{R}, x \in \Omega
$$


Substitute this inequality in the energy functional,

$$
\begin{aligned}
I_{(a, b, \lambda)}(u) & =\frac{a}{2}\|u\|^{2}+\frac{b}{4}\|u\|^{4}-\frac{\lambda}{p} \int_{\Omega}|u|^{p} d x-\int_{\Omega} G(x, u) d x \\
& \leq\left(\frac{a}{2}+C\right)\|u\|^{2}+\frac{b}{4}\|u\|^{4}-\frac{\lambda}{p} \int_{\Omega}|u|^{p} d x+C\|u\|^{\gamma},
\end{aligned}
$$

for all $u \in H_{0}^{1}(\Omega)$. Noticing that $1<p<2$ and $\lambda>0$, the existence of $s_{0}$ for each nonzero $u \in H_{0}^{1}(\Omega)$ follows by comparing the exponents in the last expression.

Step 2 There exists $\rho>0$ such that, for any $0 \neq u \in H_{0}^{1}(\Omega)$ satisfying $0<\|u\| \leq \rho$ and $I_{(a, b, \lambda)}(u)=0$, we have $\left.\frac{d}{d s} I_{(a, b, \lambda)}(s u)\right|_{s=1}>0$.

Recall the facts that $f(x, u)=\lambda|u|^{p-2} u+g(x, u)$, we have

$$
f(x, u) u=\lambda|u|^{p}+\mu|u|^{2}+o\left(|u|^{2}\right), \quad \text { for some } \mu \geq 0 \text {, as }|u| \rightarrow 0 \text {. }
$$

Denote by $F(x, u)=\int_{0}^{u} f(x, t) d t$, then for some $\tau \in(p, 2)$

$$
\tau F(x, u)-f(x, u) u=\lambda\left(\frac{\tau}{p}-1\right)|u|^{p}+\mu\left(\frac{\tau}{2}-1\right)|u|^{2}+o\left(|u|^{2}\right), \quad \text { as }|u| \rightarrow 0 .
$$

Since $\lambda>0$, there exists a $\delta>0$ small enough, such that

$$
f(x, u) u>0 \quad \text { and } \quad \tau F(x, u)-f(x, u) u \geq 0, \quad \forall 0<|u| \leq \delta, x \in \Omega
$$

Therefore there exist $\gamma_{1} \in\left(2,2^{*}\right)$ and $C>0$ such that

$$
\tau F(x, u)-f(x, u) u \geq-C|u|^{\gamma_{1}}, \quad \forall u \in \mathbb{R}, x \in \Omega .
$$

Let $0 \neq u \in H_{0}^{1}(\Omega)$ be such that $I_{(a, b, \lambda)}(u)=0$, then by (4.1) we have

$$
\begin{aligned}
\left.\frac{d}{d s} I_{(a, b, \lambda)}(s u)\right|_{s=1} & =a\|u\|^{2}+b\|u\|^{4}-\int_{\Omega} f(x, u) u d x-\tau I_{(a, b, \lambda)}(u) \\
& =a\left(1-\frac{\tau}{2}\right)\|u\|^{2}+b\left(1-\frac{\tau}{4}\right)\|u\|^{4}+\int_{\Omega}(\tau F(x, u)-f(x, u) u) d x \\
& \geq a\left(1-\frac{\tau}{2}\right)\|u\|^{2}+b\left(1-\frac{\tau}{4}\right)\|u\|^{4}-C\|u\|^{\gamma_{1}} .
\end{aligned}
$$

For $\|u\| \ll 1$, the quadratic term dominates the last expression. Thus there is a $\rho>0$ such that

$$
\left.\frac{d}{d s} I_{(a, b, \lambda)}(s u)\right|_{s=1}>0, \quad \text { for } u \text { satisfying } I_{(a, b, \lambda)}(u)=0,0<\|u\| \leq \rho .
$$

\section{Claim:}

$$
I_{(a, b, \lambda)}(s u)<0, \quad \text { for } s \in(0,1), \quad I_{(a, b, \lambda)}(u)<0, \quad 0<\|u\| \leq \rho .
$$


Indeed, if $\|u\| \leq \rho$ and $I_{(a, b, \lambda)}(u)<0$ then there is a $\tau \in(0,1)$ such that $I_{(a, b, \lambda)}(s u)<0$ for $s \in(1-\tau, 1)$ by the continuity of $I_{(a, b, \lambda)}$. Suppose that there is a $s_{0} \in(0,1-\tau]$ such that $I_{(a, b, \lambda)}\left(s_{0} u\right)=0$ and $I_{(a, b, \lambda)}(s u)<0$ as $s_{0}<s<1$. Set $u_{0}=s_{0} u$, then by (4.2) we have

$$
\left.\frac{d}{d s} I_{(a, b, \lambda)}\left(s u_{0}\right)\right|_{s=1}>0
$$

But using $I_{(a, b, \lambda)}(s u)-I_{(a, b, \lambda)}\left(s_{0} u\right)<0$ we get

$$
\left.\frac{d}{d s} I_{(a, b, \lambda)}(s u)\right|_{s=s_{0}}=\left.\frac{d}{d s} I_{(a, b, \lambda)}\left(s u_{0}\right)\right|_{s=1} \leq 0
$$

this is a contradiction. The claim holds.

Step 3 Now we define a mapping $T: B_{\rho}(0) \rightarrow[0,1]$ as

$$
T(u)= \begin{cases}1, & \text { for } u \in B_{\rho}(0) \text { with } I_{(a, b, \lambda)}(u) \leq 0, \\ s, & \text { for } u \in B_{\rho}(0) \text { with } I_{(a, b, \lambda)}(u)>0, I_{(a, b, \lambda)}(s u)=0, s<1\end{cases}
$$

and if $I_{(a, b, \lambda)}(u)>0$ then there exists a unique $T(u) \in(0,1)$ such that

$$
\begin{aligned}
& I_{(a, b, \lambda)}(T(u) u)=0, \quad I_{(a, b, \lambda)}(s u)<0, \quad \forall s \in(0, T(u)), \\
& I_{(a, b, \lambda)}(s u)>0, \quad \forall s \in(T(u), 1) .
\end{aligned}
$$

Using (4.2) and (4.4), by the implicit function theorem the mapping $T$ is continuous in $u$. Define a mapping $\eta:[0,1] \times B_{\rho}(0) \rightarrow B_{\rho}(0)$ by

$$
\eta(s, u)=(1-s) u+s T(u) u, \quad \text { for } s \in(0,1), u \in B_{\rho}(0),
$$

then the mapping $\eta$ is a continuous deformation from $\left(B_{\rho}(0), B_{\rho}(0) \backslash\{0\}\right)$ to $\left(B_{\rho}(0) \cap\right.$ $\left.I_{(a, b, \lambda)}^{0}, B_{\rho}(0) \cap I_{(a, b, \lambda)}^{0} \backslash\{0\}\right)$, where $I_{(a, b, \lambda)}^{0}=\left\{u \in H_{0}^{1}(\Omega): I_{(a, b, \lambda)}(u) \leq 0\right\}$. Since $B_{\rho}(0) \backslash\{0\}$ is contractible, by the homotopy invariance of homology group, we get

$$
\begin{aligned}
C_{\ell}\left(I_{(a, b, \lambda)}, 0\right) & =H_{\ell}\left(B_{\rho}(0) \cap I_{(a, b, \lambda)}^{0}, B_{\rho}(0) \cap I_{(a, b, \lambda)}^{0} \backslash\{0\}\right) \\
& \cong H_{\ell}\left(B_{\rho}(0), B_{\rho}(0) \backslash\{0\}\right)=0, \quad \ell \in \mathbb{N} .
\end{aligned}
$$

The proof is completed.

It is worth to point out that both $(G C 1)$ and $(G C 3)$ satisfy the growth condition in the above lemma. In the proof, the condition $1<p<2$ plays an essential role.

Lemma 4.2 Assume that $g$ satisfies $\left(f_{0}\right)$ and $g(x, u)=O(|u|)$ as $|u| \rightarrow 0$, then, for any $a>0$, $b \geq 0,1<p<2$ and $\lambda<0$ we have

$$
C_{\ell}\left(I_{(a, b, \lambda)}, 0\right)=\delta_{\ell, 0} \mathbb{F} \quad \ell \in \mathbb{N} .
$$

Proof We only need to prove that $u=0$ is a local minimizer of $I_{(a, b, \lambda)}$ in the $H_{0}^{1}(\Omega)$ topology. 
First we show that $u=0$ is a local minimizer of $I_{(a, 0, \lambda)}$ in the $C_{0}^{1}(\bar{\Omega})$ topology. Indeed, there exist $\delta>0$ and $C>0$ such that

$$
G(x, u) \leq C|u|^{2}, \quad \text { for }|u| \leq \delta, x \in \Omega
$$

Then, for $u \in C_{0}^{1}(\bar{\Omega})$ with $|u|_{\infty} \leq \delta$, we have

$$
\begin{aligned}
I_{(a, 0, \lambda)}(u) & =\frac{a}{2}\|u\|^{2}-\frac{\lambda}{p} \int_{\Omega}|u|^{p} d x-\int_{\Omega} G(x, u) d x \\
& \geq \frac{a}{2}\|u\|^{2}-\frac{\lambda}{p} \int_{\Omega}|u|^{p} d x-C \int_{\Omega}|u|^{2} d x \\
& \geq\left(\frac{-\lambda}{p}-C|u|_{\infty}^{2-p}\right) \int_{\Omega}|u|^{p} d x \\
& \geq 0
\end{aligned}
$$

provided $|u|_{\infty}^{2-p} \leq \frac{-\lambda}{p C}$. Now, using [1] we know that $u=0$ is also a local minimizer of $I_{(a, 0, \lambda)}$ in $H_{0}^{1}(\Omega)$ topology.

Moreover, since $b \geq 0$, we also know that $u=0$ is a local minimizer of $I_{(a, b, \lambda)}$ in $H_{0}^{1}(\Omega)$ topology. The proof is completed.

The proof of Theorem 1.2 will be separated into two parts, according to the sign of $\lambda$.

Proof of Theorem 1.2 (i) Case 1: $\lambda>0, m \geq \frac{1}{2}(1-\operatorname{sgn}(\lambda))=0$. Using $\left(f_{0}\right)$ and $(G C 3)$, Lemmas 3.1 and 3.3 give the existence of a Gromoll-Meyer pair $\left(W, W_{-}\right)$for $I_{(0, b, 0)}$ at 0 such that

$$
C_{m}\left(I_{(0, b, 0)}, 0\right)=H_{m}\left(W, W_{-}\right) \neq 0 \text { for some } m \geq 0 \text {. }
$$

For any $v \in H_{0}^{1}(\Omega)$, we have

$$
\left\langle I_{(a, b, \lambda)}{ }^{\prime}(u), v\right\rangle=a \int_{\Omega} \nabla u \nabla v d x+b\|u\|^{2} \int_{\Omega} \nabla u \nabla v d x-\lambda \int_{\Omega}|u|^{p-2} u v d x-\int_{\Omega} f(x, u) v d x,
$$

which implies that

$$
\begin{aligned}
& \left\|I_{(a, b, \lambda)}-I_{(0, b, 0)}\right\|_{C^{1}(W)}=\left\|I_{(a, b, \lambda)}-I_{(0, b, 0)}\right\|_{C(W)}+\left\|I_{(a, b, \lambda)}^{\prime}-I_{(0, b, 0)}^{\prime}\right\|_{C(W)} \\
& =\sup _{u \in W}\left|I_{(a, b, \lambda)}(u)-I_{(0, b, 0)}(u)\right|+\sup _{u \in W\|\nu\| \leq 1} \sup _{\| \nu}\left|\left\langle I_{(a, b, \lambda)}^{\prime}(u)-I_{(0, b, 0)}^{\prime}(u), v\right\rangle\right| \\
& \leq C a+C \lambda+C a \sup _{u \in W} \sup _{\|\nu\| \leq 1}\|u\|\|v\|+C \lambda \sup _{u \in W\|\nu\| \leq 1} \sup _{\|u\|^{p-1}\|v\|} \\
& \leq C a+C \lambda .
\end{aligned}
$$

Then, for any $\varepsilon>0$, there is $\beta>0$ such that

$$
\left\|I_{(a, b, \lambda)}-I_{(0, b, 0)}\right\|_{C^{1}(W)}<\varepsilon, \quad \text { for } 0<a, \lambda<\beta,
$$

which implies that $\left(W, W_{-}\right)$is still a Gromoll-Meyer pair for $I_{(a, b, \lambda)}$ with the critical set

$$
S_{[a, b, \lambda]}=W \cap K_{I_{(a, b, \lambda)}}, \quad \text { for } 0<a, \lambda<\beta .
$$


Therefore, for $0<a, \lambda<\beta$, using (4.5), we have

$$
C_{m}\left(I_{(a, b, \lambda)},\left[S_{[a, b, \lambda]}\right]\right)=H_{m}\left(W, W_{-}\right) \neq 0, \quad \text { for some } m \geq 0 \text {. }
$$

However, Lemma 4.1 gives

$$
C_{\ell}\left(I_{(a, b, \lambda)}, 0\right)=0, \quad \forall \ell \in \mathbb{N} \text {. }
$$

The remaining part of the proof can be carried out in a similar way to Theorem 1.1.

(i) Case 2: $\lambda<0, m \geq \frac{1}{2}(1-\operatorname{sgn}(\lambda))=1$. Using $\left(f_{0}\right)$ and (GC3), Lemmas 3.1 and 3.3 show that

$$
C_{m}\left(I_{(0, b, 0)}, 0\right) \neq 0 \quad \text { for some } m \geq 1 \text {. }
$$

Since $\lambda<0$, Lemma 4.2 gives

$$
C_{l}\left(I_{(a, b, \lambda)}, 0\right)=\delta_{l, 0} \mathbb{F} .
$$

Now, applying the arguments in the proof of Theorem 1.1 again, (4.6) and (4.7) contradict with each other. Then the equation has at least one nontrivial solution.

(ii) The proof is similar to (i), here we omit it.

\section{Proof of Theorem 1.3}

Lemma 5.1 Under the assumptions of Theorem 1.3, we have

$$
C_{\ell}\left(I_{(a, b, \lambda)}, 0\right)=\delta_{\ell, 0} \mathbb{F} .
$$

Proof By $\left(f_{0}\right)$ and (GC3), there exists $\gamma \in\left(4,2^{*}\right)$ such that

$$
|G(x, u)| \leq C\left(|u|^{4}+|u|^{\gamma}\right), \quad \forall u \in \mathbb{R}, x \in \Omega
$$

which implies that

$$
\begin{aligned}
I_{(a, b, \lambda)}(u) & =\frac{a}{2}\|u\|^{2}+\frac{b}{4}\|u\|^{4}-\frac{\lambda}{p} \int_{\Omega}|u|^{p} d x-\int_{\Omega} G(x, u) d x \\
& \geq \frac{a}{2}\|u\|^{2}-C|\lambda|\|u\|^{p}-C\|u\|^{4}-C\|u\|^{\gamma} \\
& \geq 0
\end{aligned}
$$

provided $2 \leq p<6$ and $\|u\| \geq 0$ small enough. Then we know that $u=0$ is a local minimizer of $I_{(a, b, \lambda)}$ in $H_{0}^{1}(\Omega)$ topology. The proof is completed.

Proof of Theorem 1.3 Since $g$ satisfies (GC3) for some $m \geq 1$, from Lemmas 3.1 and 3.3, we know that $u=0$ is an isolated critical point of $I_{(0, b, 0)}$, and there exists a Gromoll-Meyer pair $\left(W, W_{-}\right)$for $I_{(0, b, 0)}$ at 0 such that

$$
C_{m}\left(I_{(0, b, 0)}, 0\right)=H_{m}\left(W, W_{-}\right) \neq 0, \quad \text { for some } m \geq 1 \text {. }
$$


For any $\varepsilon>0$, using $2 \leq p<6$ and

$$
\begin{aligned}
& \left\|I_{(a, b, \lambda)}-I_{(0, b, 0)}\right\|_{C^{1}(W)}=\left\|I_{(a, b, \lambda)}-I_{(0, b, 0)}\right\|_{C(W)}+\left\|I_{(a, b, \lambda)}^{\prime}-I_{(0, b, 0)}^{\prime}\right\|_{C(W)} \\
& =\sup _{u \in W}\left|I_{(a, b, \lambda)}(u)-I_{(0, b, 0)}(u)\right|+\sup _{u \in W} \sup _{\|\nu\| \leq 1}\left|\left\langle I_{(a, b, \lambda)}^{\prime}(u)-I_{(0, b, 0)}^{\prime}(u), v\right\rangle\right| \\
& \leq C a+C|\lambda|+C a \sup \sup \|u\|\|v\|+C|\lambda| \text { sup sup }\|u\|^{p-1}\|v\| \\
& u \in \mathbb{W}\|v\| \leq 1 \quad u \in \mathbb{W}\|v\| \leq 1 \\
& \leq C a+C|\lambda|,
\end{aligned}
$$

we know that there is $\beta>0$ such that

$$
\left\|I_{(a, b, \lambda)}-I_{(0, b, 0)}\right\|_{C^{1}(W)}<\varepsilon, \quad \text { for } 0<a,|\lambda|<\beta,
$$

which implies that $\left(W, W_{-}\right)$is still a Gromoll-Meyer pair for $I_{(a, b, \lambda)}$ with the critical set

$$
S_{[a, b, \lambda]}=W \cap K_{I_{(a, b, \lambda)}}, \quad \text { for } 0<a,|\lambda|<\beta .
$$

Therefore, for $0<a,|\lambda|<\beta$, using (5.1), we have

$$
C_{m}\left(I_{(a, b, \lambda)},\left[S_{[a, b, \lambda]}\right]\right)=H_{m}\left(W, W_{-}\right) \neq 0, \quad \text { for some } m \geq 1 \text {. }
$$

On the other hand, Lemma 5.1 gives

$$
C_{\ell}\left(I_{(a, b, \lambda)}, 0\right)=\delta_{\ell, 0} \mathbb{F} \text {. }
$$

Thus Eq. (1.1) has at least one nontrivial solution.

\section{Acknowledgements}

The authors would like to express their sincere thanks to Professor Su Jiabao for his valuable suggestions, and to the reviewers for their helpful advices and comments.

\section{Funding}

This paper was supported by KZ202010028048, KM201710009012, NSFC $(11771302,11671026,11601353)$ and the funds of NCUT (110052971921/102, XN140/010, XN137/002/006).

\section{Abbreviations}

Not applicable.

Availability of data and materials

Not applicable.

\section{Competing interests}

The authors declare that they have no competing interests.

\section{Authors' contributions}

MS initiated this research and was a major contributor in writing the manuscript. YC revised the statements of Corollary 1.1, Theorem 1.3 and presented their proofs more accurately. RT substantively revised this manuscript. All authors read and approved the final manuscript.

\section{Author details}

${ }^{1}$ College of Sciences, North China University of Technology, 100144, Beijing, China. ${ }^{2}$ School of Mathematical Sciences, Capital Normal University, 100048, Beijing, China.

\section{Publisher's Note}

Springer Nature remains neutral with regard to jurisdictional claims in published maps and institutional affiliations. 


\section{References}

1. Brezis, H., Nirenberg, L.: $H^{1}$ versus $C^{1}$ local minimizers. C. R. Acad. Sci., Sér. 1 Math. 317, 465-472 (1993)

2. Chang, K.-C.: Infinite Dimensional Morse Theory and Multiple Solution Problems. Birkhäuser, Boston (1993)

3. Chang, K.-C.: Methods in Nonlinear Analysis. Springer, Berlin (2005)

4. Chang, K.-C., Ghoussoub, N.: The Conley index and the critical groups via an extension of Gromoll-Meyer theory. Topol. Methods Nonlinear Anal. 7, 77-93 (1996)

5. Chang, K.-C., Wang, Z.-Q.: Notes on the bifurcation theorem. J. Fixed Point Theory Appl. 1, 195-208 (2007)

6. Chen, C., Huang, J., Liu, L.: Multiple solutions to the nonhomogeneous p-Kirchhoff elliptic equation with concave-convex nonlinearities. Appl. Math. Lett. 26, 754-759 (2013)

7. Chen, C., Kuo, Y., Wu, T.: The Nehari manifold for a Kirchhoff type problem involving sign-changing weight functions. J. Differ. Equ. 250, 1876-1908 (2011)

8. Cheng, B., Wu, X.: Existence results of positive solutions of Kirchhoff problems. Nonlinear Anal. 71, 4883-4892 (2009)

9. Colasuonno, F., Pucci, P.: Multiplicity of solutions for $p(x)$-polyharmonic elliptic Kirchhoff equations. Nonlinear Anal. 74 5962-5974 (2011)

10. Duan, L., Huang, L.H.: Infinitely many solutions for sublinear Schrödinger-Kirchhoff-type equations with general potentials. Results Math. 66, 181-197 (2014)

11. Iannizzotto, A., Papageorgiou, N.: Existence and multiplicity results for resonant fractional boundary value problems. Discrete Contin. Dyn. Syst., Ser. S 11(3), 511-532 (2018). https://doi.org/10.3934/dcdss.2018028

12. Jiu, Q., Su, J.: Existence and multiplicity results for Dirichlet problems with $p$-Laplacian. J. Math. Anal. Appl. 281, 587-601 (2003)

13. Kirchhoff, G.: Mechanik. Teubner, Leipzig (1883)

14. Lei, C.-Y., Liu, G.-S., Guo, L.-T.: Multiple positive solutions for a Kirchhoff type problem with a critical nonlinearity. Nonlinear Anal., Real World Appl. 31, 343-355 (2016)

15. Li, A., Su, J.: Existence and multiplicity of solutions for Kirchhoff-type equation with radial potentials in $\mathbb{R}^{3}$. Z. Angew. Math. Phys. 66, 3147-3158 (2015)

16. Li, L., Zhong, X.: Infinitely many small solutions for the Kirchhoff equation with local sublinear nonlinearities. J. Math. Anal. Appl. 435, 955-967 (2016)

17. Liang, Z., Li, F., Shi, J.: Positive solutions to Kirchhoff type equations with nonlinearity having prescribed asymptotic behavior. Ann. Inst. Henri Poincaré, Anal. Non Linéaire 31, 155-167 (2014)

18. Lions, J.L.: On some questions in boundary value problems of mathematical physics. In: Contemporary Developments in Continuum Mechanics and Partial Differential Equations. Proc. Internat. Sympos., Inst. Mat. Univ. Fed. Rio de Janeiro, Rio de Janeiro. North-Holland Math. Stud., vol. 30, pp. 284-346. North-Holland, Amsterdam (1978)

19. Liu, J., Wu, S.: A note on a class of sublinear elliptic equation (1997) preprint, Peking University

20. Mao, A., Zhang, Z.: Sign-changing and multiple solutions of Kirchhoff type problems without the P.S. condition. Nonlinear Anal. 70, 1275-1287 (2009)

21. Moroz, V:: Solutions of superlinear at zero elliptic equations via Morse theory. Topol. Methods Nonlinear Anal. 10, 387-397 (1997)

22. Nyamoradi, N., Zhou, Y.: Existence of solutions for a Kirchhoff type fractional differential equations via minimal principle and Morse theory. Topol. Methods Nonlinear Anal. 46, 617-630 (2015)

23. Perera, K., Zhang, Z.: Nontrivial solutions of Kirchhoff-type problems via the Yang index. J. Differ. Equ. 221(1), 246-255 (2006)

24. Sun, J., Liu, S.: Nontrivial solutions of Kirchhoff type problems. Appl. Math. Lett. 25, 500-504 (2012)

25. Sun, J., Tang, C.: Existence and multiplicity of solutions for Kirchhoff type equations. Nonlinear Anal. 74, 1212-1222 (2011)

26. Sun, M., Su, J., Cai, H.: Multiple solutions for the $p$-Laplacian equations with concave nonlinearities via Morse theory. Commun. Contemp. Math. 19, 1650014 (2017)

27. Tayyebi, E., Nyamoradi, N.: Existence of nontrivial solutions for Kirchhoff type fractional differential equations with Liouville-Weyl fractional derivatives. Nonlinear Anal. 2018, Article ID 19 (2018)

28. Wang, L., Han, Z.: Multiple small solutions for Kirchhoff equation with local sublinear nonlinearities. Appl. Math. Lett. 59, 31-37 (2016)

29. Xiang, M., Zhang, B., Guo, X.: Infinitely many solutions for a fractional Kirchhoff type problem via fountain theorem. Nonlinear Anal. 120, 299-313 (2015)

30. Yang, Y., Zhang, J.: Nontrivial solutions of a class of nonlocal problems via local linking theory. Appl. Math. Lett. 23 , 377-380 (2010)

31. Zhang, B., Bisci, G.M., Xiang, M.: Multiplicity results for nonlocal fractional $p$-Kirchhoff equations via Morse theory. Topol. Methods Nonlinear Anal. 49, 445-461 (2017)

32. Zhang, Z., Perera, K.: Sign changing solutions of Kirchhoff type problems via invariant sets of descent flow. J. Math Anal. Appl. 317, 456-463 (2006) 